\title{
Transatlantica
}

Revue d'études américaines. American Studies Journal

1 | 2017

Morphing Bodies: Strategies of Embodiment in Contemporary US Cultural Practices

\section{Compte-rendu de « Black Dolls, la collection Deborah Neff »}

Nora Philippe, commissaire de l'exposition et cinéaste. Deborah Willis, conseillère scientifique, professeure et directrice du Département Image et Photographie de la Tisch School of the Arts à l'Université de New York (NYU)

\section{Emmanuel Falguières}

\section{(2) OpenEdition}

12 Journals

Édition électronique

URL : https://journals.openedition.org/transatlantica/8539

DOI : $10.4000 /$ transatlantica. 8539

ISSN : $1765-2766$

Éditeur

Association française d'Etudes Américaines (AFEA)

Référence électronique

Emmanuel Falguières, "Compte-rendu de «Black Dolls, la collection Deborah Neff » », Transatlantica [En ligne], 1 | 2017, mis en ligne le 29 novembre 2018, consulté le 20 mai 2021. URL : http:// journals.openedition.org/transatlantica/8539; DOI : https://doi.org/10.4000/transatlantica.8539

Ce document a été généré automatiquement le 20 mai 2021.

\section{(c) $($ ) $\odot$ (8Y NC ND}

Transatlantica - Revue d'études américaines est mise à disposition selon les termes de la licence Creative Commons Attribution - Pas d'Utilisation Commerciale - Pas de Modification 4.0 International. 


\section{Compte-rendu de « Black Dolls, la collection Deborah Neff»}

Nora Philippe, commissaire de l'exposition et cinéaste. Deborah Willis, conseillère scientifique, professeure et directrice du Département Image et Photographie de la Tisch School of the Arts à l'Université de New York (NYU)

\section{Emmanuel Falguières}

1 Parmi les quelque 400 poupées de la collection Deborah Neff, près de deux cents sont mises en espace à La maison rouge. À ce corpus de poupées « noires », toutes fabriquées aux États-Unis entre 1840 et 1940, vient s'ajouter soixante-quinze photographies également issues de cette collection ainsi que quelques éléments exogènes : deux quilts, un diorama de portraits photographiques d'Africaines-Américaines anonymes ainsi qu'un film documentaire réalisé par la cinéaste et commissaire d'exposition Nora Philippe. La maison rouge et les Éditions Fage publient également un volumineux catalogue d'exposition bilingue anglais-français. En écho à l'exposition, plusieurs évènements sont organisés hors des murs de la galerie afin de constituer un véritable " périmètre vivant $»^{1}$ autour des poupées. Parmi ceux-ci, un colloque au Quai Branly a réuni universitaires et artistes autour un dialogue élargi, historiquement et géographiquement, à la question des poupées à celle des corps noirs (Stolz 2018).

2 Le projet d'exposition a pour source la découverte par Nora Philippe du catalogue de la première et unique exposition consacrée aux poupées de la collection privée de Deborah Neff à San Diego en 2015 (Maresca 2015). Nora Philippe, cinéaste formée à l'histoire de l'art et à la muséologie, décide alors de rencontrer la collectionneuse : un projet de film documentaire autour des poupées naît de cette première entrevue. Rapidement, le besoin de montrer ces objets s'impose et prend alors forme le projet d'exposition à La maison rouge. Du livre au film, du film à l'exposition, le projet scientifique et esthétique de l'exposition «Black Dolls» est empreint d'interdisciplinarité. Fondée par la corporalité des poupées, l'exposition est une occasion de produire un ensemble de discours sur ces objets et plus largement sur l'expérience des Africaines-Américaines entre 1850 et 1950. 
Cette exposition place ces objets à l'intersection de trois grands champs qui ont connu ces dernières années une forte croissance dans le monde universitaire: les études africaines-américaines, celles sur le genre et l'histoire matérielle. Prises dans ces questionnements, les poupées noires forment un sujet d'études encore à la marge, que l'exposition permet de mettre en avant. Si l'évènement n'a pas pour vocation de proposer une «thèse " arrêtée sur ces poupées, il ouvre la voie à de futurs travaux, notamment autour de l'histoire des luttes africaines-américaines, depuis les résistances au système esclavagiste du XIX ${ }^{\text {ème }}$ siècle jusqu'aux actions pour les droits civiques des années 1960.

4 La maison rouge accueille ces œuvres dans deux grandes salles principales, auxquelles s'adjoint une salle de projection dédiée au film documentaire. Dès l'entrée, le spectateur est confronté à un double travail de décodage, à une double « lecture ». Il lui faut déchiffrer d'une part les citations tirées de textes de femmes africainesaméricaines d'hier et d'aujourd'hui qui s'affichent sur les murs blancs de la fondation et d'autre part les corps matériels des objets présentés.

Illustration 1 : Auteure inconnue, Femme aux yeux bleus et bottines rouges, États-Unis, fin du XIXe siècle

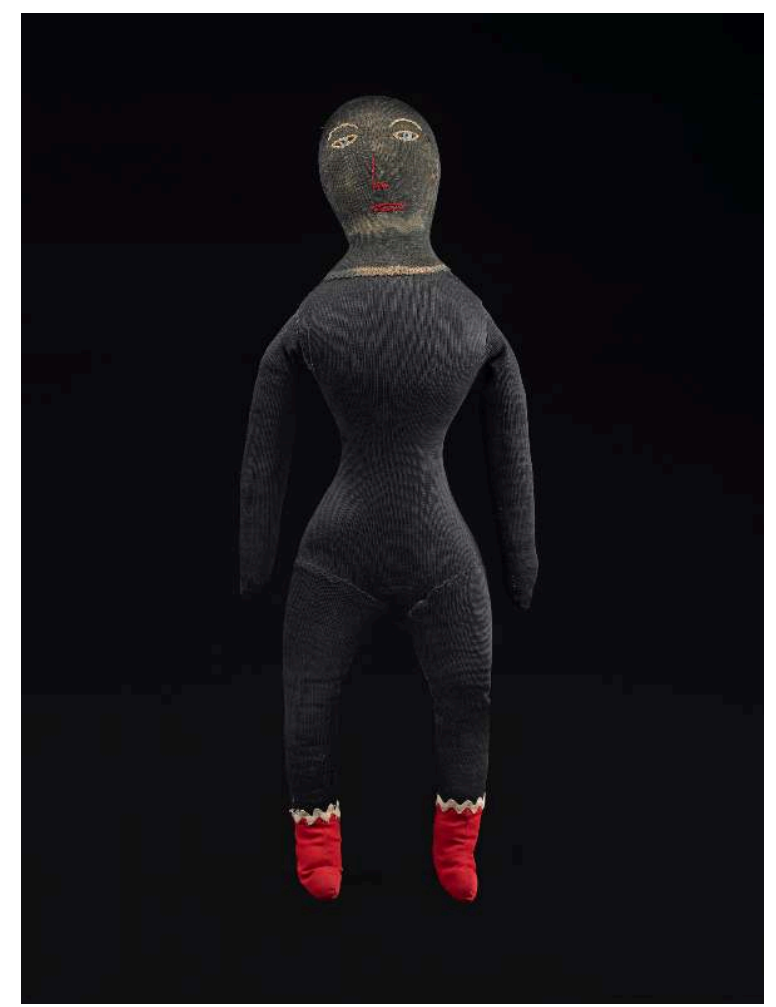

Collection Deborah Neff, photographie de Ellen McDermott, New York (Philippe et al. 2018, couverture).

La citation suivante est placée en regard :

«Comment la créativité de la femme noire a-t-elle pu rester vivante, année après année, siècle après siècle, alors que pendant la plus grande partie du temps que les Noirs ont passé en Amérique, il leur a été interdit de lire et d'écrire? Et la liberté de peindre, de sculpter, de s'ouvrir l'esprit par l'action qui n'existait pas.» Alice Walker, In Search of our Mother's Gardens, 1983. 
Le corps de la poupée est implicitement posé comme une réponse aux questions d'Alice Walker. Si chaque spectateur ou spectatrice saisit les implications des citations en fonction de ses connaissances, l'appréhension sensible de la poupée, quant à elle, résiste. Entourés de contextualisations suggérées par les notices d'expositions, les coupures de presse et les citations (sur l'histoire des femmes, de l'esclavage, de la ségrégation, ou de l'espace domestique), ces corps en matériaux composites, issus de procédés de récupération et de réutilisation, sont autant de questions sans réponses.

5 Il aura fallu attendre qu'une juriste blanche originaire de Long Island se mette à collectionner personnellement au gré de voyages et des rencontres ces poupées, pour que puisse se constituer les preuves tangibles de la continuité de cette pratique, et qu'un corpus apparaisse. Deborah Neff n'a pas constitué sa collection sur des critères prédéterminés mais s'est laissée guider par une émotion esthétique. Lors d'une brocante à Atlanta, la collectionneuse, qui s'ignore encore, rencontre sa première poupée. Elle est avant tout frappée par sa simplicité, sa composition de « chutes de cuir, de clous et de bois " et sa non-conformité aux représentations stéréotypées du corps africain-américain de son époque. Deborah Neff a décidé que son corpus n'irait pas plus loin que la période de la seconde guerre mondiale. Ce choix vient d'une décision intuitive qui mêle une réalité matérielle, (moins de poupées sont faites à la main après 1940), et un jugement esthétique: "[les poupées postérieures] peuvent être "mignonnes" mais pas expressives. Elles ne suscitent pas en moi l'émotion que provoqua cette première poupée de cuir.» (Philippe et al. 2018, 24) Les poupées constituent donc un ensemble visuel hétérogène : petites ou grandes, habillées ou non, réalistes ou stylisées, en tissus malléables ou en bois dur, chaque poupée est unique et les types sont nombreux. Nulle "école» ni "période» n'émane de l'ensemble. Toutefois, la démarche intime de Déborah Neff n'est pas sans cohérence interne. De l'accumulation naît une définition du type de poupées noires retenues : elles sont faites à la main, avec des matériaux de couleur sombre (les poupées blanches peintes en noir ne sont pas retenues) et le plus souvent fabriquées hors d'un circuit commercial (pour autant qu'on puisse en juger). Ces éléments ne statuent pas sur l'identité des auteurs ou des auteures qui pourraient être blanches ou noires mais l'exposition présume d'une production a priori majoritairement féminine et africaine-américaine. Une collection constituée dans un cadre plus traditionnellement académique aurait donné lieu à bornage strict. La collection Neff, elle, offre de rafraichissantes exceptions. Par exemple, les poupées de l'artiste Mae Rowe datent des années 1970 et les poupées de Leo Moss furent fabriquées avec une visée commerciale. Ces deux cas détonnent dans l'exposition mais permettent des chemins de traverses qui, sans perdre le spectateur, l'invite à s'interroger sur les liens entre poupées vernaculaires et créations d'artistes mais aussi entre pratiques informelles et commerce. Ces achoppements dans la cohérence du corpus agissent comme des rappels du thème au cœur de la collection : l'intime des pratiques domestiques.

\section{Le pluriel et le singulier : poupées noires}

6 Le spectateur entre dans l'exposition par un couloir rappelant les pratiques domestiques enfantines de la poupée. Dans une sorte d'antichambre, quelques poupées rudimentaires sont intégrées à de très petits décors, découpés dans un carton uni, façon «maison de poupées». Ces simples scénographies rappellent la fonction 
première des poupées, le jeu d'enfant. Une porte, une fenêtre, un meuble, et une dramaturgie prend forme, rappellant ces poupées à une vie imaginaire qui fut sans nul doute leur lieu primordial.

7 À ce court vestibule fait suite la première salle. Elle est dédiée aux poupées elles-mêmes et propose une très impressionnante mise en espace et en lumière, d'un peu plus de 150 spécimens. Les poupées sont présentées en groupes, articulés autour de grands thèmes - le portrait, le cadeau, la reprise. Cet effet de groupe est mis en tension avec le choix d'une présentation individualisée des poupées qui souligne leur statut d'œuvre unique: chaque création est posée sur un piédestal en bois individuel. Ces supports permettent un jeu scénographique sur la hauteur à laquelle sont vues les poupées. Alors que certaines poupées sont placées à hauteur d'enfant, d'autres s'élèvent au niveau du regard d'un adulte. Ce jeu sur les hauteurs, associé aux effets de groupes, transforme ces figures humaines en petits groupements, ou «bosquets» qui habitent l'espace, longent les murs ou surgissent au centre de la pièce. Au-dessus de ces "bosquets ", des explications dans les deux langues sont inscrites aux murs et viennent donner à chaque groupe un éclairage scientifique ou un ton particulier.

Illustration 2 : Scénographie de la salle d'exposition 1 de « Black Dolls » à La maison rouge

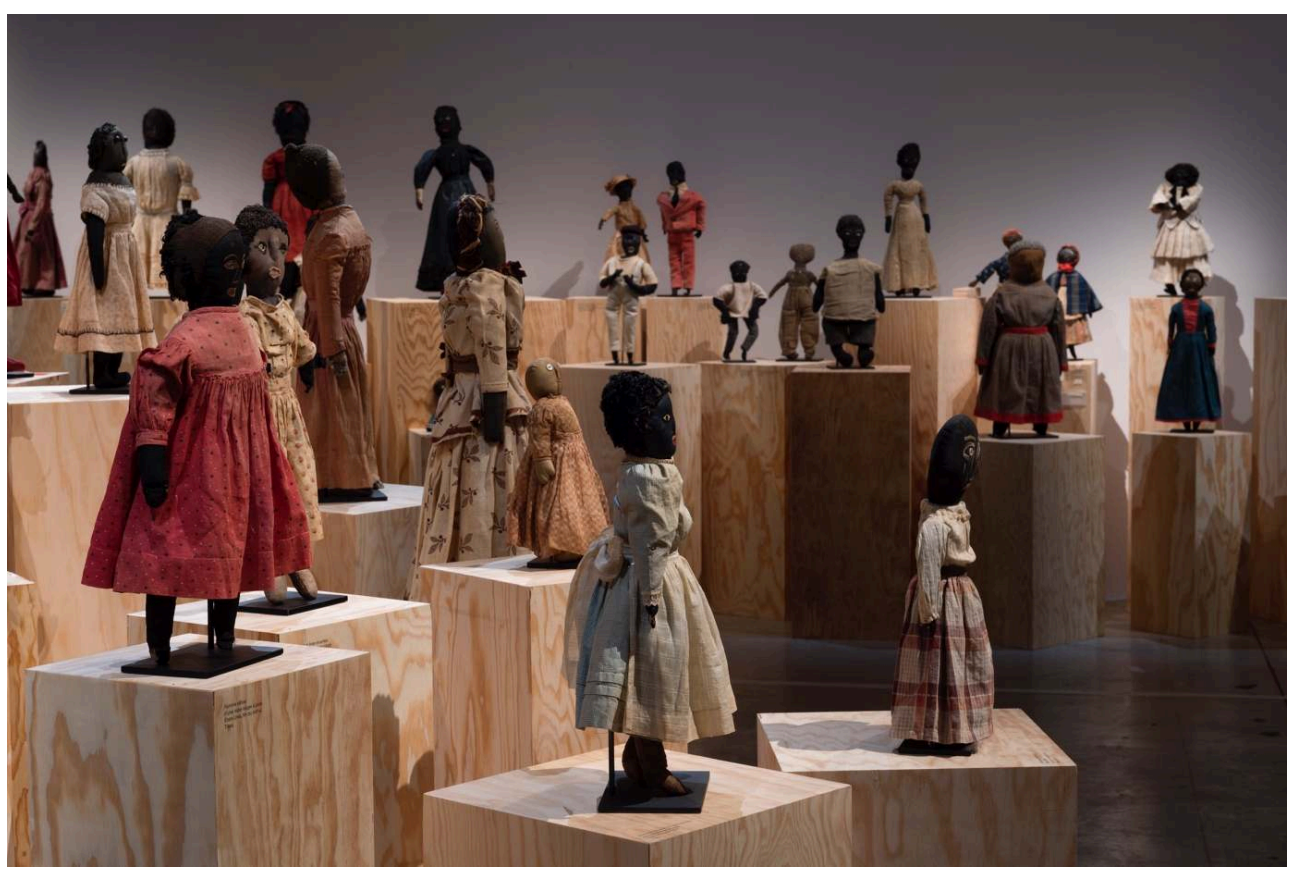

Photographie de Marc Domage, 2018.

Plus que les jeux d'enfants, l'exposition s'attache à mettre en avant les auteures inconnues de ces poupées et leur mode de fabrication. Comment parler de cet «avant » des poupées?

Deux grands axes se dessinent grâce au léger appareil critique qui accompagne l'exposition. Premièrement, les poupées noires sont de silencieux témoins de l'histoire. L'exposition lie explicitement la production des poupées à l'histoire des AfricainsAméricains au cours du XIX ${ }^{e}$ et du premier $\mathrm{XX}^{\mathrm{e}}$ siècle, elle associe à la création de ces modestes objets une résistance continue à l'ordre social. 
Dans un second temps, la poupée noire est présentée comme une preuve de l'existence d'un art populaire de la communauté africaine-américaine. L'épineux problème de la datation des poupées est en partie fondé par l'anonymat de leurs auteures. Le style du vêtement, le type de tissu, la forme des points de coutures ainsi que les teintures renseignent sur la provenance historique et géographique. Toutefois, la question de l'origine se heurte à la vie matérielle des poupées qui, dans leur majorité, se transforment au fur et à mesure des années et des usages. Fabriquée pour un ou une enfant, la poupée noire entre dans une circulation aléatoire. Elle peut être reprisée par une autre femme, donnée à un autre enfant de la famille, changer de vêtements bien des années plus tard, etc. Ainsi, les générations se succèdent et les poupées vivent. Leur origine "s'épaissit » et s'éloigne résolument de la notion d'auteure individuelle pour prendre une dimension collective au grès des circonstances et des propriétaires successifs.

11 Les expressions sur les visages des poupées sont extrêmement diverses. Si certaines possèdent un sourire brodé à même le tissu, d'autres ont au contraire des larmes dessinées à même la joue. La plupart proposent une stylisation qui permet au spectateur d'aujourd'hui (et à l'enfant d'hier) d'y projeter ses propres émotions. Cette expression épurée peut successivement prendre les oripeaux de la colère, de la tendresse ou de la joie. L'énigme de ce visage ambivalent, changeant à chaque jeu, constitue aussi l'efficacité de la poupée.

Illustration 3 : Auteure inconnue, Femme portant une tunique avec motif cachemire, États-Unis, premier quart du $\mathrm{XX}^{\mathrm{e}}$ siècle

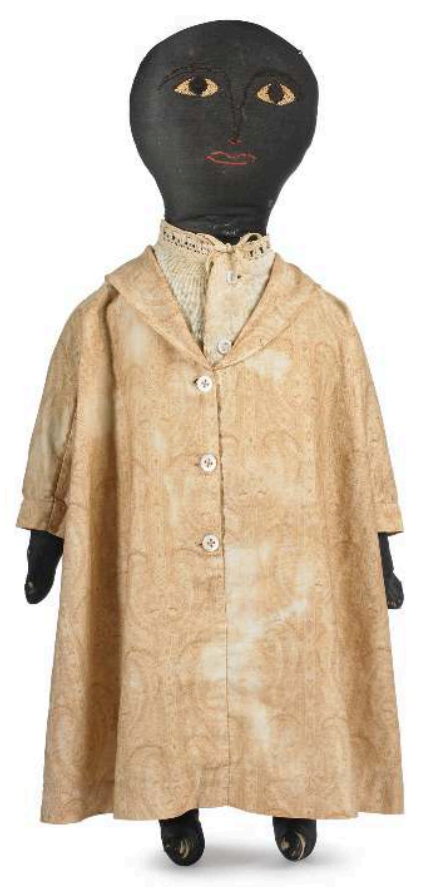

Coton, 66 × 26 × 8,5 cm, collection Deborah Neff. [Woman in Paisley Coat Dress. NEFF 183], photographie de Ellen McDermott, New York. (Philippe et al. 2018, 15). 
12 En présentant une longue série s'étirant depuis le milieu du XIX ${ }^{\mathrm{e}}$ siècle jusqu'au XX siècle, l'exposition propose une contre-histoire des représentations racistes du corps noir mais aussi de la «negro doll " commerciale et industrielle qui apparaît dans les premières décennies du $\mathrm{XX}^{\mathrm{e}}$ siècle et produite par des Africains-Américains pour des Africains-Américains. Alors que l'espace de la salle est tout entier dédié à la poupée noire domestique, des reproductions de publicités pour ces poupées « nègres » au début $\mathrm{du} \mathrm{XX}^{\mathrm{e}}$ siècle viennent clore le parcours du spectateur. Ces publicités suggèrent le racisme sous-tendant les représentations des corps noirs au plus fort des lois Jim Crow ${ }^{2}$. Ce pas de côté dans l'exposition met en exergue les transformations conjointes des modes de productions domestiques et ceux de la consommation de masse. C'est au sein de ces transformations que s'inventent certaines résistances aux représentations racistes telles que celles portées par les poupées noires de la collection. L'arc historique tracé par l'ensemble des poupées ouvre alors de nouvelles perspectives pour saisir les relations d'interdépendance entre production, consommation et représentations culturelles dans un contexte de seconde révolution industrielle. L'exposition insiste à juste titre dans son appareil critique sur le contexte historique global des poupées, à savoir les relations interraciales dans un pays d'abord esclavagiste puis ségrégué. C'est dans ce contexte de violence à la fois raciale et genrée que ces poupées sont cousues et utilisées. Au cœur du quotidien enfantin, les tensions visibles et invisibles d'un pays en conflit surgissent dans des objets façonnés par des mains qui vivent les maltraitances et les discriminations. Toutefois, cette analyse des violences dans laquelle sont fabriquées ces poupées mériterait d'être affinée à la fois en termes de géographie et de périodes historiques. Ces différenciations viendraient souligner la multiplicité et l'hétérogénéité des significations et des rôles que portent et jouent les corps des poupées.

\section{Usages, images et questionnements : photographies et topsy-turvies}

13 La seconde salle est consacrée à l'usage des poupées par la présentation sur trois murs de photographies de la collection Déborah Neff, tandis que le centre de la pièce est occupé par les poupées topsy-turvy, qui constituent peut-être la série d'objets la plus déroutante de cette exposition. 
Illustration 4 : Scénographie de la salle d'exposition 2 de « Black Dolls » à La maison rouge

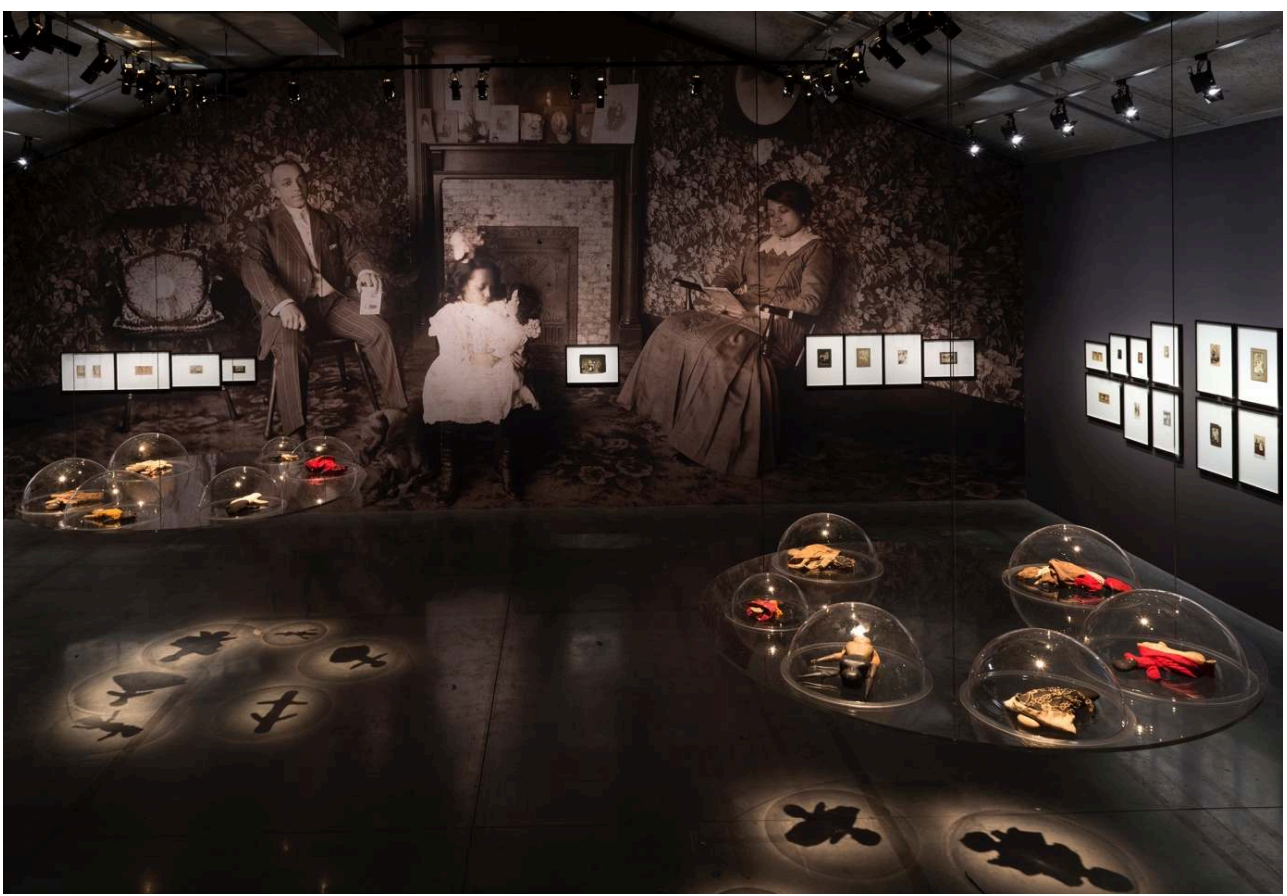

Photographie de Marc Domage, 2018.

La série de photographies est une étude du motif de l'enfant à la poupée. Les clichés représentent des enfants blancs avec des poupées noires, des enfants noirs avec des poupées blanches, des enfants noirs avec des poupées noires ; toutes et tous posent. De moment photographique en moment photographique, les attitudes se répondent alors que les contextes socio-historiques varient : l'enfant blanche de classe aisée posant avec sa poupée noire dans un daguerréotype du milieu du XIX siècle (illustration 5) côtoie un cliché de 1911 en Arkansas d'un groupe mixte au sein duquel une AfricaineAméricaine adulte tient une poupée noire qu'elle vend sous l'appellation "negro fancy work» (Philippe et al. 2018, 220-21). Qu'est-ce qui appartient au regard du ou de la photographe? Qu'est-ce qui relève de la spécificité du modèle qui pose? Qu'est-ce qui découle de relations sociales plus larges? Chacun de ces éléments s'entremêle dans les photographies, rendant ces scènes uniques presque incomparables. Pourtant, là encore, un fil commun traverse la diversité et les photographies déroulent la question commune de la race de la poupée. Les questions, et non les réponses, viennent lier les images entre elles et mettent les visiteurs en tension. Entre révélation et doute, ils contemplent la relation entre poupée et enfant se décliner au fil de la série. Informé par les quelques explications qui y sont données, le spectateur est invité à s'interroger sur la ligne de couleur entre la poupée inerte et l'enfant vivant dans ces « moments-faitsobjets » que sont les photographies.

L'unique daguerréotype présenté (illustration 5) peut servir d'exemple. L'enfant pose, sagement assise, et regarde directement le portraitiste, tenant dans ses bras une grande poupée noire de genre masculin. La chaise, la robe de la petite fille, ses cheveux peignés, mais aussi la monture dorée ouvragée du daguerréotype, le daguerréotype luimême, sont autant d'éléments qui désignent un milieu social aisé. La poupée quant à elle, bien que de bonne facture et coiffé d'un couvre-chef, dénote une pratique impliquant des matériaux modestes, des codes de représentations moins réalistes. La 
datation entre 1855 et 1865 met le cliché au cœur du conflit entre le Nord et le Sud où l'abolition de l'esclavage n'est plus seulement une question économique mais met en jeu l'équilibre de la nation. Si la photographie est prise dans un des états du Nord, elle recèlerait alors au moins deux aspects. Elle serait à la fois un portrait de l'enfant chéri affublé de son jouet préféré et une déclaration de soutien à la cause abolitionniste, notamment si l'achat de la poupée a servi à lever des fonds pour l'effort de guerre. A l'inverse, cette photographie pourrait tout aussi bien être considérée comme une appropriation quotidienne d'un travail d'esclave ou de domestique, et le signe de la présence invisible d'une nourrice africaine-américaine. Quelle que soit la narration choisie, un corps noir masculin stylisé fait irruption dans l'imagerie du portrait d'une jeune fille blanche de bonne famille, et vient interroger notre compréhension du fonctionnement des représentations raciales au cœur de l'environnement familial.

Illustration 5 : Auteure inconnue, vers 1855-1865

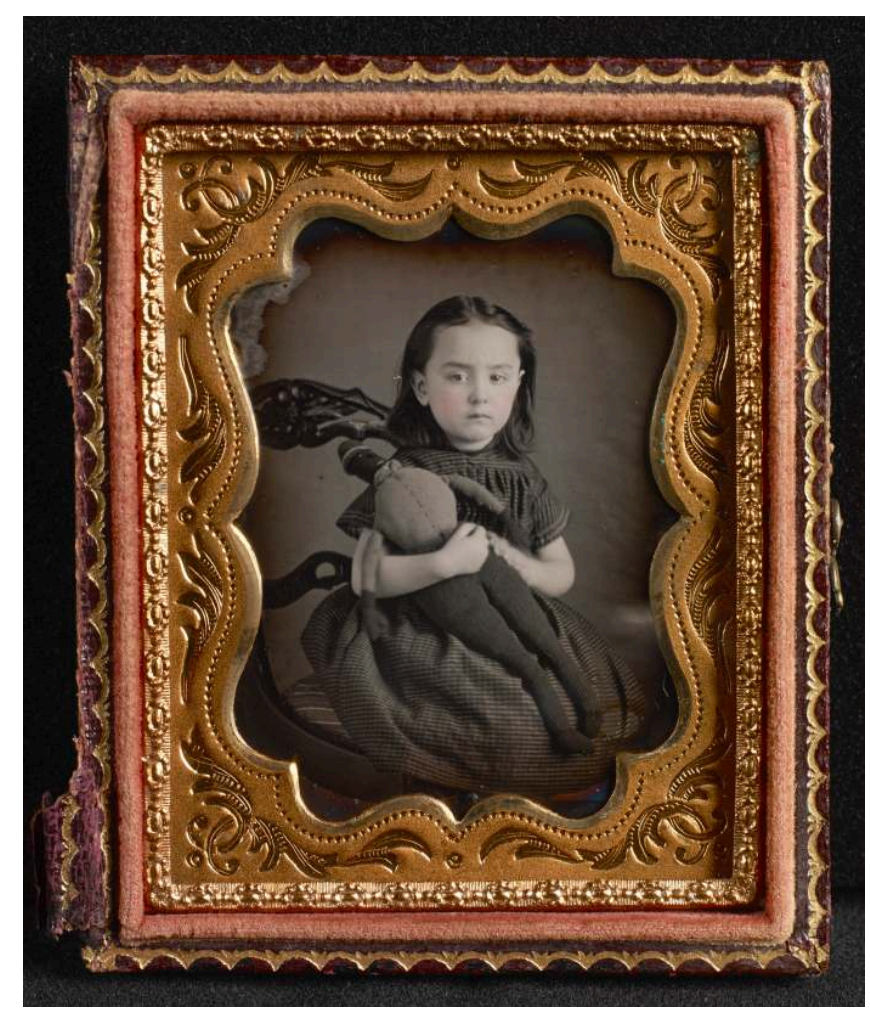

Inscription sur la tranche : « Jean Frantz », daguerréotype colorié, 6,3 x $5 \mathrm{~cm}$, collection Deborah Neff [NEFF 10001]. Reproduction photographique de Ellen McDermott, New York (Philippe et al. 2018, 189).

Attiré par un jeu d'ombres au sol, le spectateur découvre au centre de la salle des topsyturvies étendues sur de grands plateaux circulaires en plexiglas. Ces poupées doubles sont dites « réversibles ». Attachées par le torse, une moitié est en tissu sombre, l'autre en tissu clair. La majorité des poupées est affublée d'une jupe que l'enfant peut retourner. Il ou elle joue avec une poupée noire, la retourne et trouve une poupée blanche. Et vice-versa. Probablement apparue dans les plantations sudistes du début du $\mathrm{XIX}^{\mathrm{e}}$ siècle, ces poupées gagnèrent en popularité tout au long du premier $\mathrm{XX}^{\mathrm{e}}$ siècle (Jarboe 2015). Au vu de la production théorique des études sur la race et l'identité africaine-américaine, la poupée topsy-turvy frappe par sa pertinence quant aux 
questionnements à la fois sur la double identité de la communauté noire américaine mais aussi le crosslining entre communautés noires et blanches aux États-Unis.

Pourtant, l'objet reste mystérieux. On imagine aisément le déploiement de significations multiples auxquelles ces poupées ont pu donner lieu tant chez les enfants que chez les adultes. L'exposition, dans un court texte présentant les topsy-turvies, fait état des pistes de la recherche actuelle. Le catalogue poursuit un peu plus loin ces hypothèses travaillées par quelques chercheurs et chercheuses aux États-Unis (Philippe et al. 2018, 67-76). Les archives permettant d'appuyer les différentes interprétations sont rares. Ces topsy-turvies servaient-elles à expliquer l'identité des enfants métis issus dans de nombreux cas de viols? Étaient-elles utilisées par les nourrices africainesaméricaines pour raconter des histoires aux enfants blancs dont elles avaient la charge (Wallace-Sanders 2009, 38-52) ? Permettaient-elles aux enfants esclaves de rejouer leurs fréquents compagnonnages avec un ou une enfant blanche ${ }^{3}$ ? Enfin, constituaient-elles la subversion d'un interdit fait à un enfant blanc ou noir de jouer avec une poupée d'une "race " différente? Les chantiers restent ouverts et demandent un dialogue entre histoire sociale, histoire culturelle et histoire matérielle. Ces hypothèses auraient tout intérêt à se décliner autour de variables géographique, sociale et historique. Ces interprétations apparaissent parfois comme concurrentes mais il n'est pas exclu qu'une même poupée fabriquée pour un usage précis ai pu être utilisée par les générations suivantes dans un autre. Les circulations des poupées et de leurs usages ne cessent de créer pour les spectateurs une série d'apories qui, loin de représenter des échecs, sont autant d'occasions de saisir les complexités de ces objets.

Illustration 6 : Auteure inconnue, Poupée réversible minimale, États-Unis vers 1920-1930

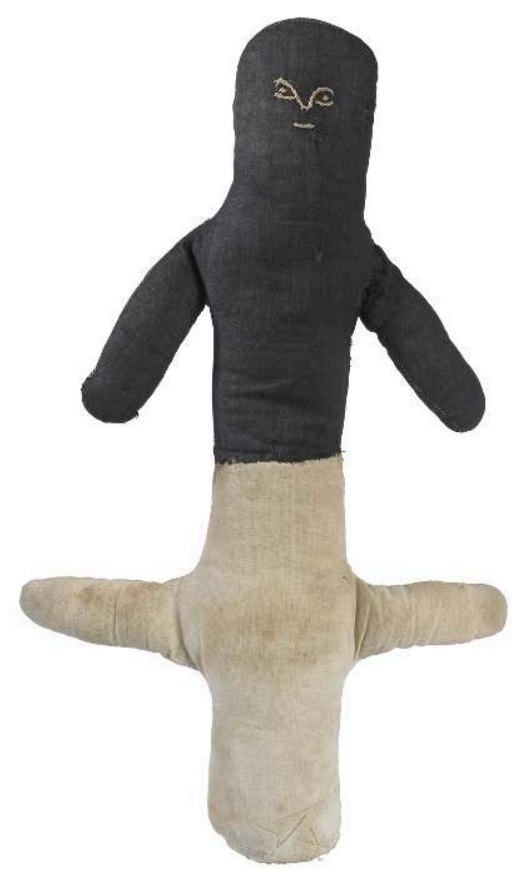

Coton, 35,5 × 24,1 × 3,8 cm, collection Deborah Neff, [Minimal Topsy-Turvy, NEFF 241], photographie de Ellen McDermott, New York (Philippe et al. 2018, 180-81). 
Les mouvements des poupées noires à travers différentes classes sociales et communautés raciales sont soulignés dans l'article que Madelyne Shaw consacre dans le catalogue d'exposition aux problèmes posés par la matérialité des poupées noires de la collection Deborah Neff.

Il est clair que les poupées noires étaient fabriquées par des femmes et des enfants de couleur aussi bien que par des femmes blanches et qu'elles s'adressaient indistinctement à des enfants noirs ou blancs. Les personnes qui les confectionnaient ou jouaient avec représentaient toute l'échelle sociale, de très pauvre à très riches, en passant par tout l'éventail des classes moyennes (Philippe et al. 2018, 85).

Si les photographies nous montrent précisément la présence de poupées noires indistinctement entre les mains d'enfants blancs ou noirs, l'exposition dans son ensemble s'intéresse relativement peu à cette question de l'objet partagé et en fait une production essentiellement ancrée dans la communauté africaine-américaine. Dans cette exposition, les poupées sont a priori des œuvres de femmes africainesaméricaines et, à ce titre, entrent en résonnance avec le double combat du féminisme et des luttes d'émancipation raciale. Ce choix structure l'ensemble de la mise en espace de la commissaire Nora Philippe et constitue le parti-pris de son film documentaire, dont une version est présentée dans l'exposition.

\section{Des voix pour des poupées ? Film documentaire}

Le visiteur est invité dans une dernière salle au sous-sol. Après être passé devant deux petits écrans diffusant des clichés de «femmes ayant pu fabriquer ou jouer avec ces poupées ", le spectateur découvre un film documentaire projeté sur grand écran. D’une vingtaine de minutes, Like Dolls, I'll Rise n'est pas une présentation de l'histoire de la collection Neff mais plutôt un essai cinématographique de donner à ces objets silencieux que sont les poupées noires une voix possible. La réalisatrice Nora Philippe fait lire à des Africaines-Américaines d'aujourd'hui des textes écrits par des AfricainesAméricaines du $\mathrm{XIX}^{\mathrm{e}}$ et du $\mathrm{XX}^{\mathrm{e}}$ siècle. Le film obéit à un double régime visuel : des séquences en studio avec les poupées, alternées de séquences de lecture des textes. A ces deux types d'image, s'ajoutent ponctuellement quelques archives, notamment des coupures de presse et un court extrait d'un film d'archive.

Filmées par le documentariste Emmanuel Gras, les séquences de mise en scène des poupées rappellent la tentative des cinéastes Chris Marker et Alain Resnais dans leur court-métrage Les statues meurent aussi (1953). Censuré dans sa version intégrale jusqu'en 1964, Les statues meurent aussi est une critique acerbe du colonialisme européen, et dénonce le vol et le recel d'objets africains exposés avec complaisance dans de grands musées belges, anglais et français. L'hommage formel au film de Marker et Resnais se traduit par un filmage sur fond noir, en studio, des poupées qui deviennent de véritables "objets-acteurs ». Ici, une image en couleur et une mise en scène joueuse des poupées remplacent l'ascèse du noir et blanc du film de 1953. Objets de projection par excellence, les poupées se transforment au cours du film: d'abord taquines, elles se font lieux de recueillement d'une histoire douloureuse. 
Illustration 7 : photogramme du film de Nora Philippe, Like Dolls, I'Il Rise

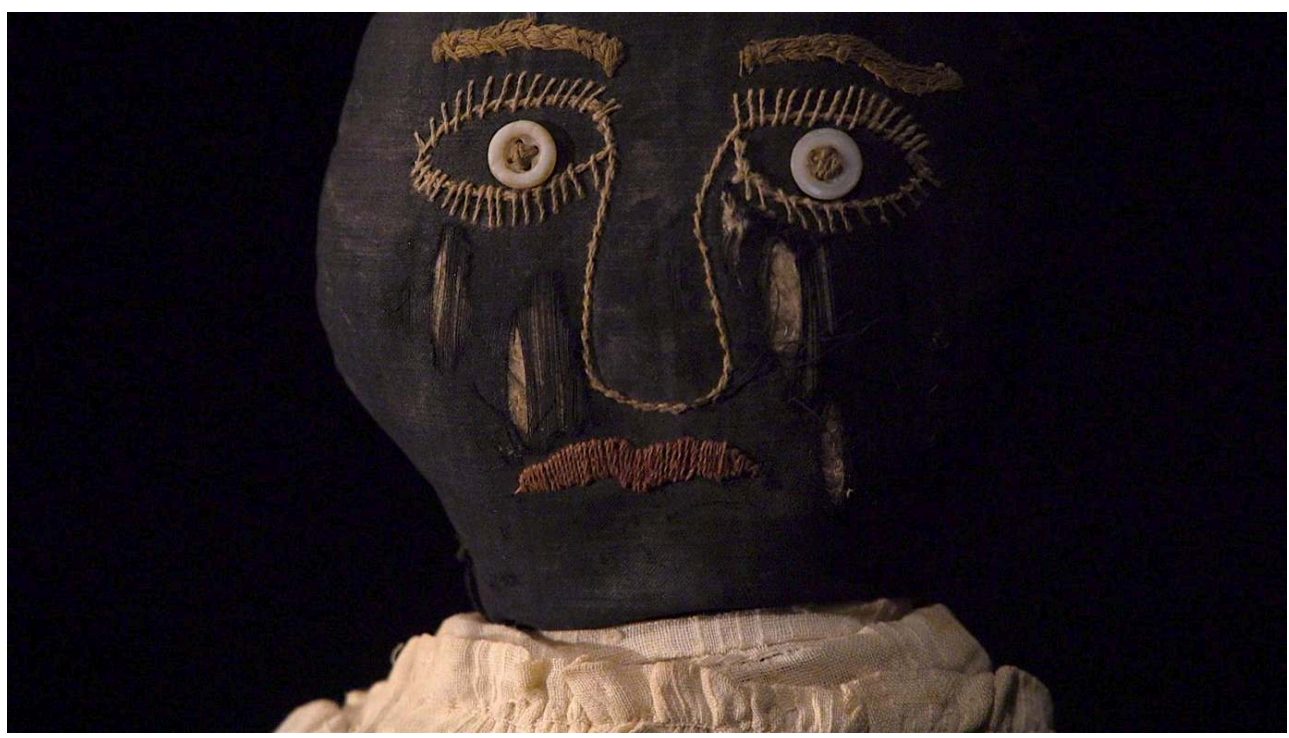

Image d'Emmanuel Gras. Les films de l'air, 2018, avec la permission des Films de l'air.

Cette transformation est incarnée dans le film par la lecture à voix haute des textes choisis. Nora Philippe a proposéà un groupe de femmes noires américaines une série de textes connus écrits par des Africaines-Américaines entre 1840 et aujourd'hui, de Sojourner Truth à Maya Angelou. Chaque lectrice ${ }^{4} \mathrm{a}$ alors choisi le texte qu'elle désirait porter à l'écran et le livre, en anglais, face caméra. Ces paroles, douloureuses mais aussi militantes, deviennent à la fois les voix possibles de celles qui fabriquèrent ou jouèrent avec les poupées mais aussi, plus métaphoriquement, les voix des combats pour sortir de l'invisibilité sociale, dans une nation où les Africaines-Américaines furent deux fois discriminées. Cette lutte contre la disparition vient rappeler la survivance des fragiles morceaux de tissus que sont in fine les poupées. Les textes comme les poupées sont têtus.

Illustration 8 : photogramme du film de Nora Philippe, Like Dolls, I'll Rise

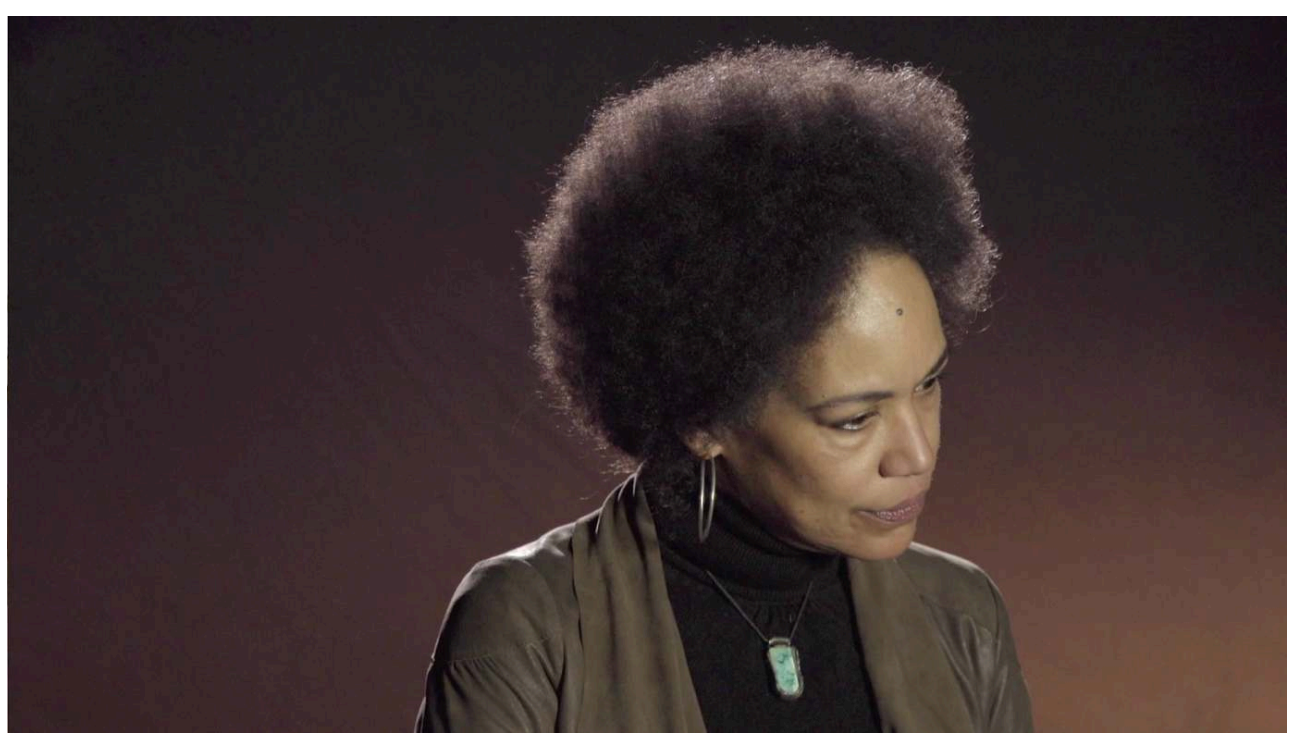

Image d'Emmanuel Gras. Les films de l'air, 2018, avec la permission des Films de l'air. 
«Black Dolls, la collection Deborah Neff» est un point de départ, l'ouverture d'une porte vers une nouvelle compréhension des expériences africaines-américaines. Pour pouvoir s'approcher de ces "vécus", il aura fallu une série de hasards: une collectionneuse touchée par une émotion esthétique loin de "son" histoire, une cinéaste retrouvant son expertise de commissaire d'exposition, une fondation parisienne qui accepte d'exposer ces objets fragiles - la liste des rencontres circonstancielles est longue. À travers ces contingences, les poupées survivent. Reste à espérer que l'articulation des traces de cet art populaire et vernaculaire aux histoires et récits américains plus traditionnels ne fasse que commencer.

Ce compte-rendu est en partie issu d'un entretien avec la commissaire d'exposition Nora Philippe le 2 mai 2018. Qu'elle en soit remerciée.

\section{BIBLIOGRAPHIE}

BEECHER STOWE Harriet, Uncle Tom's Cabin; or, Life Among the Lowly, Boston and Cleveland, John P. Jewett and Jewett, Proctor \& Worthington, 1852.

JARBOE Julian K, « The Racial Symbolism of the Topsy-Turvy Doll », The Atlantic, 2015, https:// www.theatlantic.com/technology/archive/2015/11/the-racial-symbolism-of-the-topsy-turvydoll/416985/ (page consultée le 14 mai 2018).

KAHN Eve M., 2015, « A Love for Vintage Dolls That Never Grows Old », The New York Times, 2015, https://www.nytimes.com/2015/02/06/arts/design/a-love-for-vintage-dolls-that-never-growsold.html (page consultée le 2 mai 2018).

MARESCA Frank, Black Dolls, Santa Fe, Radius Books, 2015.

PHILIPPE Nora, NEFF Deborah, BERNSTEIN Robin, RowE Nellie Mae, WILLIS Deborah et WILLIAMS Patricia, Black Dolls, la collection Deborah Neff, Paris, Fage Éditions et La maison rouge, 2018.

STOLZ Joëlle, « "Black Dolls”, une exposition qui libère la mémoire douloureuse des poupées noires », Le Monde.fr, 2018, http://www.lemonde.fr/afrique/article/2018/03/07/blacks-dolls-uneexposition-qui-libere-la-memoire-douloureuse-des-poupees-noires_5267243_3212.html (page consultée le 2 mai 2018).

WALKER Alice, In Search of Our Mothers' Gardens: Womanist Prose. San Diego, Harcourt Brace Jovanovic, 1983.

WALLACE-SANDERS Kimberly, Mammy: A Century of Race, Gender, and Southern Memory. Ann Arbor, University of Michigan Press, 2009.

YAEGER Lynn, « The Black Dolls on Showcase at a Parisian Gallery Have a Poignant Story to Tell ", Vogue, 2018, https://www.vogue.com/article/la-maison-rouge-black-dolls, (page consultée le 2 mai 2018). 
« Black dolls, material culture and Afro-American resistance and representations (1840-1940) », Colloquium, Calenda, 2018, https://calenda.org/431827 (page consultée le 15 mai 2018).

\section{Filmographie}

PHILIPPE Nora, Like Dolls, I'll Rise, Paris, Les films de l'air, 2018.

SCHOESER Jean-Nicolas, Interviews Deborah Neff, Nora Philippe, Deborah Willis, Paris, La maison rouge, 2018.

\section{NOTES}

1. Expression de la commissaire Nora Philippe. Entretien avec l'auteur du 2 mai 2018.

2. Les lois Jim Crow actaient les principes de ségrégation entre Blancs et Noirs dans le Sud des États-Unis entre 1877 et les années 1950.

3. Pratique courante se retrouve notamment dans le classique abolitionniste La Case de l'oncle Tom, avec les personnages de « Topsy » et de « Eva » (Beecher Stowe 1852).

4. Candyce Miales, Camille Andrea Rich, Catherine Zlatkovic, Raina Lampkins Fielder et ses filles.

\section{RÉSUMÉS}

Du 23 février au 20 mai 2018, l'espace d'exposition parisien La maison rouge propose la première présentation hors des États-Unis de la collection privée de poupées noires de l'américaine Deborah Neff.

From February 23rd to May 20th 2018, the Parisian exhibition space La maison rouge presents for the first time outside of the United States Deborah Neff private collection of black dolls.

\section{INDEX}

Mots-clés : Exposition, Poupées noires, Africaines-Américaines, Histoire, Culture matérielle, Topsy-Turvy, Film documentaire, collection Deborah Neff

Keywords : Exhibition, Black dolls, African-American women, History, Material culture, TopsyTurvy, Documentary film, Deborah Neff collection

\section{AUTEUR}

\section{EMMANUEL FALGUIÈRES}

Ecole des Hautes Etudes en Sciences Sociales 